\title{
Long-acting recombinant fusion protein linking coagulation factor IX with albumin (rIX-FP) in children
}

\section{Results of a phase 3 trial}

\begin{abstract}
Gili Kenet ${ }^{1}$; Hervé Chambost'; ${ }^{2}$ Christoph Male3; Thierry Lambert4; Susan Halimeh5; Tatiana Chernova6; Maria Elisa Mancuso7; Julie Curtin ${ }^{8,9}$; Christine Voigt ${ }^{10}$; Yanyan Li ${ }^{10}$; Iris Jacobs ${ }^{10}$; Elena Santagostino7; the PROLONG-9FP Investigator Study Group*

${ }^{1}$ The Israeli National Hemophilia Center, Sheba Medical Center, Tel Hashomer, Sackler Medical School, Tel Aviv University, Israel; ${ }^{2}$ Pediatric Hematology Oncology Department, Children Hospital La Timone, APHM, Aix Marseille University, Marseille, France; ${ }^{3}$ Department of Pediatrics, Medical University of Vienna, Vienna, Austria; ${ }^{4}$ Centre de Traitement des 'Hémophilies, Hôpital Bicetre, Paris, France; ${ }^{5} \mathrm{CRC}$ Coagulation Research Centre GmbH, Duisburg, Germany; ${ }^{6}$ Kirov Research Institute of Hematology and Blood Transfusion, Kirov, Russia; ${ }^{7}$ Angelo Bianchi Bonomi Hemophilia and Thrombosis Center, Fondazione IRCCS Ca' Granda, Ospedale Maggiore Policlinico, Milan, Italy; ${ }^{8} \mathrm{Children's} \mathrm{Hospital} \mathrm{at} \mathrm{Westmead,}$ Sydney Children's Hospital Network, Sydney, Australia; ${ }^{9}$ Discipline of Paediatrics and Child Health, University of Sydney, Australia; ${ }^{10} \mathrm{CSL}$ Behring, CRD, King of Prussia,

Pennsylvania, USA
\end{abstract}

\begin{abstract}
Summary
A global phase 3 study evaluated the pharmacokinetics, efficacy and safety of a recombinant fusion protein linking coagulation factor IX with albumin (rIX-FP) in 27 previously treated male children (1-11 years) with severe and moderately severe haemophilia B (factor IX [FIX] activity $\leq 2$ IU/dl). All patients received routine prophylaxis once every seven days for up to 77 weeks, and treated any bleeding episodes on-demand. The mean terminal half-life of rIX-FP was 91.4 hours (h), 4.3-fold longer than previous FIX treatment and clearance was $1.11 \mathrm{ml} / \mathrm{h} / \mathrm{kg}, 6.4$-fold slower than previous FIX treatment. The median (Q1, Q3) annualised spontaneous bleeding rate was 0.00 $(0.00,0.91)$ and was similar between the $<6$ years and $\geq 6$ years age groups, with a weekly median prophylactic dose of $46 \mathrm{IU} / \mathrm{kg}$. In addition, patients maintained a median trough level of $13.4 \mathrm{IU} / \mathrm{dl}$ FIX activity on weekly prophylaxis. Overall, $97.2 \%$ of bleeding episodes were
\end{abstract}

successfully treated with one or two injections of rIX-FP $(95 \% \mathrm{Cl}$ : $92 \%$ to $99 \%$ ), $88.7 \%$ with one injection, and $96 \%$ of the treatments were rated effective (excellent or good) by the Investigator. No patient developed FIX inhibitors and no safety concerns were identified. These results indicate that rIX-FP is safe and effective for preventing and treating bleeding episodes in children with haemophilia B with weekly prophylaxis. Routine prophylaxis with rIX-FP at treatment intervals of up to 14 days are currently being investigated in children with severe and moderately severe haemophilia B. Clinicaltrials.gov (NCT01662531)

\section{Keywords}

Haemophilia B, factor IX, on-demand treatment, prophylactic regimen, rIX-FP

Financial support:

This study was sponsored by CSL Behring, Marburg, Germany. Editorial assistance was provided by Meridian HealthComms, funded by CSL Behring.

Received: March 3, 2016

Accepted after minor revision: May 23, 2016

Epub ahead of print: September 1, 2016

http://dx.doi.org/10.1160/TH16-03-0179

Thromb Haemost 2016; 116: 659-668

\section{Introduction}

Haemophilia B patients are at an increased risk of bleeding due to the underlying factor IX (FIX) deficiency. In particular, severe (FIX activity $<1 \mathrm{IU} / \mathrm{dl}$ ) and moderate (1 to 5 IU/dl FIX activity) haemophilia are associated with spontaneous bleeding into joints, muscles and soft tissues that may lead to crippling arthropathy, while bleeding in the intracranial, neck/throat or gastrointestinal spaces may be life-threatening. The prevention of recurrent bleeding and joint deterioration in order to preserve normal musculoskeletal function is the goal of routine prophylactic treatment (1). Current prophylactic therapy requires frequent intravenous injection of FIX replacement product, maintaining appropriate FIX trough levels to effectively reduce the incidence of haemarthroses and other bleeding episodes. Standard half-life FIX replacement products require intravenous injections twice weekly $(2,3)$. The necessity of frequent injections creates a burden for both patients and caregivers, impacting long-term compliance (4).

Recombinant fusion protein linking recombinant coagulation factor IX with recombinant albumin (rIX-FP, CSL Behring) is produced as a single protein with a cleavable linker between FIX and albumin that is derived from the endogenous activation peptide in native FIX. rIX-FP has demonstrated improved pharmacokinetics (PK) and prolonged pharmacodynamic activity in earlier clinical trials conducted in adolescent and adult haemophilia B patients (5, 6). The improved PK profile of rIX-FP, including over five-fold 
slower clearance compared with recombinant FIX (rFIX), may allow patients to be injected less frequently while maintaining a desirable circulating FIX level high enough to minimise the occurrence of spontaneous bleeding episodes.

The aim of this study was to evaluate the PK, efficacy and safety of rIX-FP administered weekly as routine prophylaxis for the prevention of bleeding episodes in previously treated children with severe or moderately severe haemophilia B (FIX activity $\leq 2 \mathrm{IU} / \mathrm{dl}$ ). We present the results of a study that examined the efficacy and safety of a seven-day prophylaxis regimen in patients with haemophilia B.

\section{Materials and methods}

\section{Study conduct}

The study was approved by the institutional review board/ethics committee at each participating centre, registered at clinical- trials.gov (NCT01662531), and performed in accordance with good clinical practice (7), the Declaration of Helsinki and local regulatory requirements. Written informed consent was obtained from the parent/guardian of the patient and informed assent from the patient. Consent could be withdrawn at any time.

\section{Study patients}

The main criteria for patient selection were based on the Guideline on the clinical investigation of recombinant and human plasma-derived factor IX products by the Committee for Medicinal Products for Human Use (CHMP) (8). Male patients aged 0-11 years with severe or moderately severe haemophilia B (FIX activity $\leq 2 \mathrm{IU} / \mathrm{dl}$ ) who had received FIX products for $>150$ exposure days (EDs) for patients 6-11 years old, and $>50$ EDs for patients $<6$ years old, were eligible for enrolment. Patients had no confirmed personal or family history of FIX inhibitor formation or detectable inhibitors at screening.

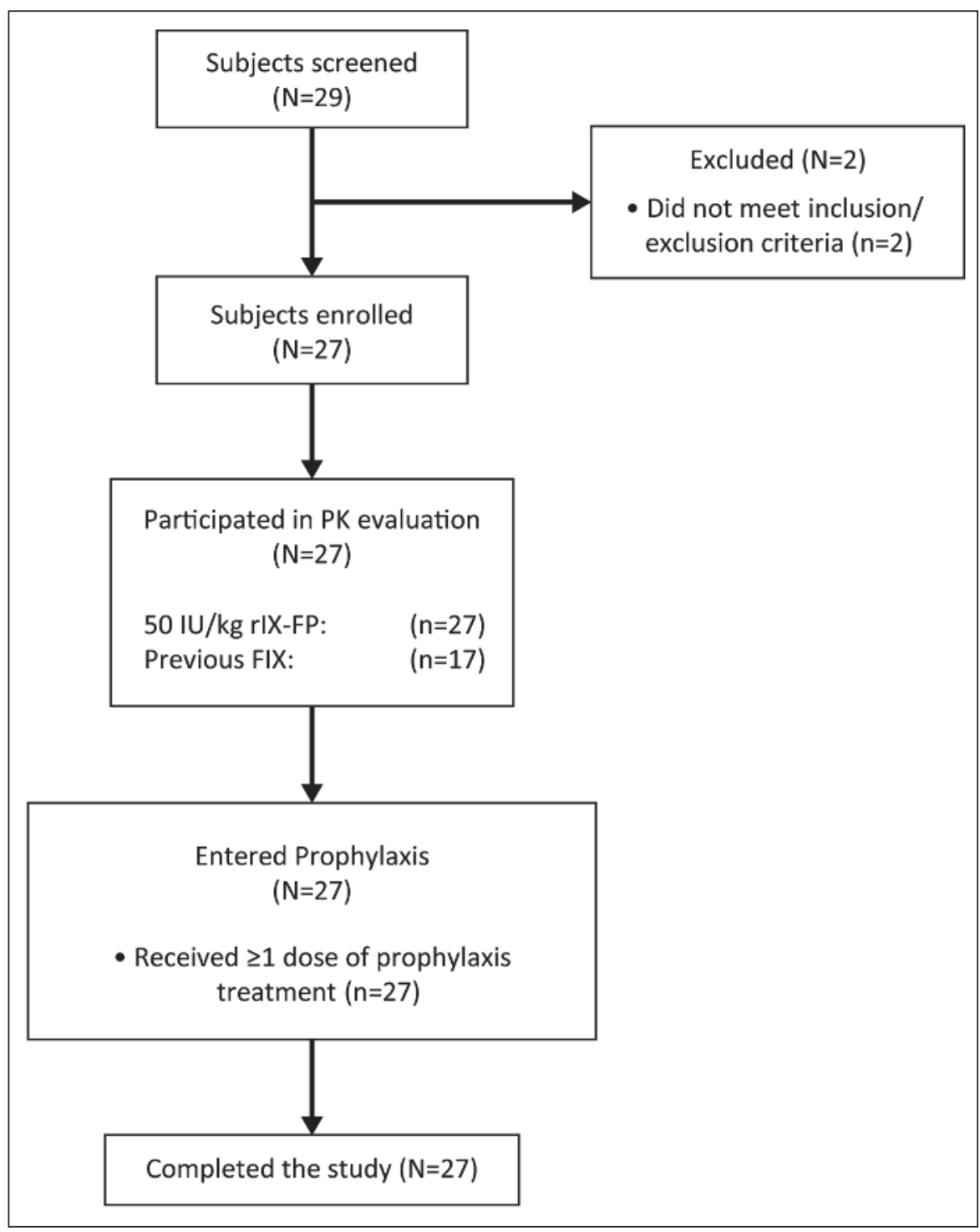

Figure 1: Patient enrolment and study flow diagram. The diagram shows the flow and disposition of patients in the study. Following screening, 27 patients were enrolled in the study. All patients participated in PK evaluation of 50 IU/kg rIX-FP. A subset of patients $(n=17)$ also participated in a PK evaluation of their previous FIX product (pdFIX or rFIX). All patients were assigned weekly prophylaxis with a dose of 35-50 IU/kg. All patients completed the study. FIX, factor IX; pdFIX, plasma-derived FIX; PK, pharmacokinetics; rFIX, recombinant FIX; rIX-FP, recombinant fusion protein linking coagulation factor IX with albumin. 
Patients with a known hypersensitivity to any FIX product or hamster protein, undergoing immunomodulating treatment or having a CD 4 cell count of $<200 / \mathrm{mm}^{3}$ were excluded. Patients with serum aspartate aminotransferase or alanine aminotransferase activity at $>5$ times the upper limit of normal were also excluded.

\section{Trial design}

This was a prospective, non-randomised, international, open-label phase 3 study, with all patients assigned to weekly prophylactic treatment ( $>$ Figure 1). Patients underwent up to one-month screening followed by active treatment for 12 months or longer. All patients participated in PK evaluation of $50 \mathrm{IU} / \mathrm{kg}$ rIX-FP at study entry. Patients also participated in PK evaluation of $50 \mathrm{IU} / \mathrm{kg}$ of previous FIX product unless PK data were available in the medical records. The patients were assigned a dose of 35-50 IU/kg rIX-FP for weekly prophylaxis, at the investigator's discretion. The dose could be adjusted higher or lower, based on bleeding phenotype, physical activity level, and clinical outcome, while maintaining a weekly treatment interval. The active treatment period for the evaluation of safety and efficacy was extended up to 18 months to allow patients to receive continuous treatment with rIX-FP until enrolment in the subsequent extension study. Efficacy and safety assessments were performed at study sites approximately every six weeks.

All bleeding episodes which occurred during the treatment period of the study were treated with rIX-FP. For the treatment of a bleeding episode, the dose was based upon the patient's PK profile, World Federation of Hemophilia guidelines and the local standard of care, with a minimum dose of 35 IU/kg rIX-FP. rIX-FP treatment was administered at home by either the patient or patient's caregiver for both routine prophylaxis and the treatment of bleeding episodes. Treatments and bleeding episodes (including causality, i.e. spontaneous, traumatic or unknown) were recorded by patients or their caregiver in an electronic diary.

Quality of life measurements were collected at screening and end of study, and activity levels were reported at every study visit.

\section{Trial objectives and outcome measures}

The primary objectives of the study were to evaluate the safety of rIX-FP, as assessed by the occurrence of inhibitors against FIX in all patients who received dose(s) of rIX-FP, and to evaluate the PK of rIX-FP. PK parameters evaluated included incremental recovery, half-life, area under the curve (AUC) and clearance of a single dose of rIX-FP.

The secondary objectives of the study were to i) evaluate the efficacy of rIX-FP to prevent bleeding episodes by weekly prophylaxis and to treat bleeding episodes, and ii) evaluate the overall clinical safety of repeated dosing of rIX-FP. The secondary efficacy endpoints were assessed by the annualised bleeding rates (ABRs) in the seven-day prophylactic regimen, the number of rIX-FP injections required to achieve haemostasis when treating bleeding episodes and the Investigator's assessment based on a four-point scale (see footnote of $>$ Table 4 for details). Safety was determined by evaluating the frequency, severity and relatedness of adverse events (AEs), development of non-inhibitory antibodies against rIX-FP and Chinese hamster ovary $(\mathrm{CHO})$ cell proteins, local tolerability, laboratory safety parameters, physical exam and vital signs.

\section{Analytical methods}

FIX activity was measured using a validated one-stage clotting method using Pathromtin SL (Siemens Healthcare Diagnostics, Marburg, Germany) as an activator reagent; rIX-FP activity determination was performed using the Behring Coagulation System (5). The results were interpreted using a reference curve, prepared from standard human plasma calibrated to the World Health Organization standard for FIX, and reported in International Units (IU). This assay was used to measure FIX activity for PK assessment of both rIX-FP and the patients' previous FIX product (plasma-derived FIX [pdFIX] or rFIX).

Inhibitors were titrated by the Bethesda method according to the Nijmegen modification (9), and a titre of $\geq 0.6$ Bethesda units was defined as a positive result.

A tiered approach to immunogenicity testing for rIX-FP was employed during the study. A direct-binding enzyme-linked immunosorbent assay (ELISA) assay was performed to detect antibodies against rIX-FP; if a positive signal was obtained, the sample was re-tested to confirm the specific antibody signal and to discriminate between antibodies against rFIX, pdFIX and albumin. A second ELISA assay was performed to test for antibodies against $\mathrm{CHO}$ cell proteins; if a positive signal was obtained, the sample was re-tested by surface plasmon resonance to confirm the specific antibody signal. All assays were performed in the central laboratory at CSL Behring, Marburg, Germany.

\section{Statistical analysis}

The efficacy and safety populations consisted of all patients who received at least one dose of rIX-FP. The sample size of the study was based on the recommendations from regulatory agencies due to the rarity of the disease. The efficacy of routine prophylaxis was examined by annualised spontaneous bleeding rate (AsBR), presented with summary statistics. In addition, the estimated AsBR (model-based AsBR) with a 95\% confidence interval (CI) based on a Poisson distribution was presented for all treatment regimens.

Treatment of minor or moderate bleeding episodes was considered successful if haemostasis was achieved with one or two injections. The proportion of bleeds treated successfully was reported with a $95 \% \mathrm{CI}$. In order to consider on-demand treatment as effective, one would expect to observe $85 \%$ of bleeding episodes being treated with one or two injections (10). Therefore, as an acceptance criterion, the lower limit of the two-sided $95 \% \mathrm{CI}$ was set at $80 \%$.

The PK analysis was performed by standard non-compartmental analysis using Phoenix WinNonlin software version 6.2 (Pharsight). Four main PK parameters (incremental recovery, terminal half-life, AUC and total body clearance normalised to body 
Table 1: Baseline demographics and patient characteristics.

\begin{tabular}{|c|c|c|c|}
\hline Characteristics & $\begin{array}{l}<6 \text { years } \\
(\mathrm{N}=12)\end{array}$ & $\begin{array}{l}6-11 \text { years } \\
(\mathrm{N}=15)\end{array}$ & $\begin{array}{l}\text { Total } \\
(\mathrm{N}=27)\end{array}$ \\
\hline Mean age (SD), years & $3.2(1.70)$ & $8.1(1.41)$ & $5.9(2.93)$ \\
\hline \multicolumn{4}{|l|}{ Race, $n(\%)$} \\
\hline White & $11(91.7)$ & $15(100.0)$ & $26(96.3)$ \\
\hline Black/African-American & $1(8.3)$ & 0 & $1(3.7)$ \\
\hline Mean weight (SD), kg & $16.0(3.81)$ & $31.4(9.73)$ & $24.6(10.87)$ \\
\hline \multicolumn{4}{|l|}{ Geographic location, n (\%) } \\
\hline Asia Pacific & 0 & $2(13.3)$ & $2(7.4)$ \\
\hline Europe & $9(75.0)$ & $11(73.3)$ & $20(74.0)$ \\
\hline Middle East & $2(16.7)$ & $2(13.3)$ & $4(14.8)$ \\
\hline North America & $1(8.3)$ & 0 & $1(3.7)$ \\
\hline \multicolumn{4}{|l|}{ Previous treatment regimen } \\
\hline On-demand & $1(8.3)$ & $2(13.3)$ & $3(11.1)$ \\
\hline Prophylaxis & $11(91.7)$ & $13(86.7)$ & $24(88.9)$ \\
\hline \multicolumn{4}{|l|}{ Previous FIX product } \\
\hline $\mathrm{rFIX}$ & $10(83.3)$ & $11(73.3)$ & $21(77.8)$ \\
\hline pdFIX & $2(16.7)$ & $4(26.7)$ & $6(22.2)$ \\
\hline \multicolumn{4}{|c|}{ Previous prophylaxis interval, $\mathrm{n}(\%)^{*}$} \\
\hline$<2$ times per week & $1(9.1)$ & $3(23.1)$ & $4(16.7)$ \\
\hline 2 times per week & $8(72.7)$ & $7(53.8)$ & $15(62.5)$ \\
\hline$\geq 3$ times per week & $2(18.2)$ & $1(7.7)$ & $3(12.5)$ \\
\hline \multicolumn{4}{|c|}{ Previous FIX weekly consumption, IU/kg } \\
\hline Mean (SD) & $138.7(123.83)$ & $80.3(47.25)$ & $107.1(93.34)$ \\
\hline Target joint $†, n(\%)$ & $2(16.7)$ & $1(6.7)$ & $3(11.1)$ \\
\hline \multicolumn{4}{|l|}{ Total ABR $\ddagger$} \\
\hline Mean (SD) & $6.5(9.98)$ & $7.4(13.56)$ & $7.0(11.89)$ \\
\hline Median (IQR) & $3.0(1.0,4.5)$ & $2.0(1.0,6.0)$ & $3.0(1.0,6.0)$ \\
\hline \multicolumn{4}{|l|}{ AsBR $\ddagger$} \\
\hline Mean (SD) & $2.8(5.11)$ & $4.9(11.74)$ & $3.9(9.18)$ \\
\hline Median (IQR) & $0.5(0.0,2.5)$ & $0.0(0.0,1.0)$ & $0.0(0.0,2.0)$ \\
\hline History of catheter use, $n(\%)$ & $2(16.7)$ & $1(6.7)$ & $3(11.1)$ \\
\hline \multicolumn{4}{|l|}{ Activity level, n (\%) } \\
\hline Sedentary & 0 & $1(6.6)$ & $1(3.7)$ \\
\hline Moderately active & $7(58.3)$ & $6(40.0)$ & $13(48.1)$ \\
\hline Vigorous & $5(41.7)$ & $8(53.3)$ & $13(48.1)$ \\
\hline
\end{tabular}

$A B R$, annualised bleeding rate; $A s B R$, annualised spontaneous bleeding rate; FIX, factor IX; IQR, interquartile range; pdFIX, plasma-derived factor IX; rIX, recombinant factor IX; SD, standard deviation. *Percentages are based on the number of patients who received prophylaxis prior to study entry. + Percentages are based on the number of patients with a target joint, defined as at least 3 spontaneous bleeding episodes in the same joint in a 6-month period (22). \#Bleeding episodes in the 12 months prior to study entry.
Table 2: Factor IX genotype of patients.

\begin{tabular}{|c|c|c|c|}
\hline \multicolumn{2}{|l|}{ Mutation* } & \multirow{2}{*}{$\begin{array}{l}\text { Previous } \\
\text { reportst }\end{array}$} & \multirow[t]{2}{*}{ Type of mutation } \\
\hline cDNA & AA/codon & & \\
\hline c.711A $>\mathrm{G}$ & Gln237Gln & 2 & Silent \\
\hline c.136delA & p.Arg46GlyfsX58 & 0 & Deletion, frameshift \\
\hline c.1279G >A & Gly427Arg & 5 & Missense \\
\hline c.1369A $>\mathrm{T}$ & Lys457Stop & 1 & Nonsense \\
\hline$c .880 C>T$ & Arg294Stop & 70 & Nonsense \\
\hline c. $676 C>T$ & Arg226Trp & 44 & Missense \\
\hline c. $226 \mathrm{G}>\mathrm{A}$ & Glu76Lys & 3 & Missense \\
\hline-- & Val377del & 6 & Deletion \\
\hline c. $128 \mathrm{G}>\mathrm{A}$ & Arg43Gln & 85 & Missense \\
\hline c. $871 \mathrm{G}>\mathrm{A}$ & Glu291Lys & 5 & Missense \\
\hline$c .830 \mathrm{~T}>\mathrm{A}$ & Val277Asp & 1 & Missense \\
\hline c.686G >T & Gly229Val & $1 \ddagger$ & Missense \\
\hline
\end{tabular}

* Nucleotide and codon numbering according to Human Genome Variation Society (HGVS) $(23,24)$, in which the first codon is the first residue (Met) of the 46-residue signal peptide/propeptide, and the first residue of the mature protein is 47 . $†$ The number of patients reporting the mutation as captured in the Factor IX Variant Database, available at www.factorix.org. (13). ‡ Report of this mutation is in the CDC Hemophilia B Mutation Project (CHBMP) F9 Database only (25).

weight) were calculated using the actual collection times, according to International Society on Thrombosis and Haemostasis recommendations $(11,12)$.

\section{Drug product}

rIX-FP was produced by expression in $\mathrm{CHO}$ cells and supplied as freeze-dried powder in single-use vials with a nominal content of $250,500,1000$ or $2000 \mathrm{IU}$ per vial. The 250,500 and $1000 \mathrm{IU}$ vials were reconstituted with $2.5 \mathrm{ml}$, and the $2000 \mathrm{IU}$ vial with $5.0 \mathrm{ml}$, of water for intravenous injection.

\section{Results}

\section{Patient characteristics}

Overall, 27 male patients with haemophilia B (FIX activity $\leq 2$ IU/ dl) from 17 sites in 10 countries provided informed consent and were enrolled and treated with rIX-FP between January 2013 and October 2014. Patient enrolment and a study flow diagram are presented in $>$ Figure 1. Enrolment was evenly distributed between the younger ( $<6$ years) and older (6-11 years) age groups, with 12 and 15 patients, respectively. Patients were between 1 and 11 years of age, with a mean of 3.2 years in the younger age group, and 8.1 years in the older age group. There were six $(50 \%)$ patients who had between 50 and 150 EDs to FIX products at study entry in the younger age group. The patient population was primarily 
from Europe (74.0\%), but included patients from North America, the Asia-Pacific region and the Middle East ( $\triangleright$ Table 1). The majority of patients were receiving prophylaxis prior to study entry, with a twice-weekly prophylactic regimen. Patients in the younger age group had a shorter prophylactic dose interval than the older age group. No patient had hepatitis B, hepatitis C or HIV. Three patients had a history of central venous access catheter use, but only one patient used a catheter during the study. Patients reported an overall high level of physical activity, with $67 \%$ of patients in the older age group playing sports on a weekly basis, including soccer.

If available in the medical records, genotype of the FIX mutation was recorded. Twelve patients had FIX mutations identified; no patients had the same mutation, as shown in $\mathbf{T a b l e} 2$. The majority of mutations identified were point (missense) mutations; one mutation (Arg294Stop) in one patient causing a large deletion has been reported to be associated with the development of inhibitors in the Factor IX Variant Database (13).

\section{Pharmacokinetics}

All patients participated in a PK assessment of rIX-FP at the start of the study, and a subset of patients participated in a PK assessment of their previous product, either rFIX or pdFIX. Overall, rIXFP demonstrated an improved PK profile compared with either rFIX or pdFIX, with an incremental recovery of approximately 1 (IU/dl)/(IU/kg) for both age groups, which was $40 \%$ higher than that of the patients' previous FIX product. In addition, rIX-FP had a half-life of 91.4 hours (h), AUC of $4894 \mathrm{IU} \cdot \mathrm{h} / \mathrm{dl}$ and clearance of $1.112 \mathrm{ml} / \mathrm{h} / \mathrm{kg}$ ( Table 3). The half-life of rIX-FP was similar between the younger and older age groups (89.6 and $92.8 \mathrm{~h}$, respectively), and much longer than that of the previous FIX products (19.9 and $17.7 \mathrm{~h}$, respectively).

After administration of a single intravenous dose of $50 \mathrm{IU} / \mathrm{kg}$ rIX-FP, plasma FIX activity was higher in both age groups com- pared with $50 \mathrm{IU} / \mathrm{kg}$ of previous FIX product. The mean FIX activity levels in all paediatric patients remained above $5 \mathrm{IU} / \mathrm{dl}$ through day 10 and above $2 \mathrm{IU} / \mathrm{dl}$ through day 14 following a dose of 50 IU $/ \mathrm{kg}$ ( Figure 2). In contrast, mean FIX activity of a single dose of either $50 \mathrm{IU} / \mathrm{kg}$ rFIX or pdFIX was below $5 \mathrm{IU} / \mathrm{dl}$ within $48 \mathrm{~h}$.

\section{Efficacy}

\section{Once-weekly prophylactic treatment with rIX-FP}

All 27 patients were on weekly prophylactic treatment for a mean of 62 weeks. The median ABRs were 0.0 and 0.78 for younger and older age groups, respectively ( $>$ Table 4 ). The median total ABR was 3.12, with similar total ABR between the two age groups. Most of the bleeding episodes reported were due to trauma, which is not unexpected for this active population, and occurred in the second half of the prophylaxis interval.

There were very few spontaneous bleeding episodes reported during the study, with the majority in the older age group ( Table

5). All occurred at least $72 \mathrm{~h}$ after the last prophylaxis dose, with the majority after $144 \mathrm{~h}$. Of the joint bleeds reported, only nine $(22 \%)$ were spontaneous; all other joint bleeds were due to trauma. Only one patient had more than one spontaneous bleed in the same joint; no patients developed a target joint during the study. In addition, nine (33\%) patients reported 22 bleeding episodes that did not require treatment: 12 were nasal bleeds, and the remaining 10 were joint and/or muscle bleeds.

There were three patients who received on-demand treatment only prior to study entry; the AsBR for these patients were markedly reduced in the study when compared with the number of spontaneous bleeding episodes reported in the 12 months prior to study entry. A similar reduction (85-94\%) in total ABR occurred with weekly treatment with rIX-FP compared with on-demand treatment with standard FIX products prior to study entry. Nevertheless, during the study, the bleeding rates among these

Table 3: Pharmacokinetic parameters of rIX-FP and previous FIX product in children with haemophilia B.

\begin{tabular}{|c|c|c|c|c|c|c|}
\hline \multirow{3}{*}{$\begin{array}{l}\text { PK parameter } \\
\text { (Unit)* }^{*}\end{array}$} & \multicolumn{3}{|c|}{ rIX-FP 50 IU/kg } & \multicolumn{3}{|c|}{ Previous FIX 50 IU/kg } \\
\hline & $\begin{array}{l}<6 \text { years } \\
(\mathrm{N}=12)\end{array}$ & $\begin{array}{l}6-11 \text { years } \\
(\mathrm{N}=15)\end{array}$ & $\begin{array}{l}\text { Total } \\
(\mathrm{N}=27)\end{array}$ & $\begin{array}{l}<6 \text { years } \\
(\mathrm{N}=8)\end{array}$ & $\begin{array}{l}6-11 \text { years } \\
(\mathrm{N}=9)\end{array}$ & $\begin{array}{l}\text { Total } \\
(\mathrm{N}=17)\end{array}$ \\
\hline & \multicolumn{6}{|c|}{ Mean (\%CV) } \\
\hline IR (IU/dl)/(IU/kg) & $0.951(21.5)$ & $1.06(22.6)$ & $1.01(22.5)$ & $0.676(20.7)$ & $0.793(29.3)$ & $0.738(26.8)$ \\
\hline$A U C_{0-\infty}(I U \cdot h / d l)$ & $4583(33.2)$ & $5123(31.4)$ & $4894(32.0)$ & $886(70.1)$ & $890(21.3)$ & $888(46.9)$ \\
\hline$t_{1 / 2}(h)$ & $89.6(12.5)$ & $92.8(20.5)$ & $91.4(17.5)$ & $19.9(40.3)$ & $17.7(25.6)$ & $18.6(33.0)$ \\
\hline $\mathrm{CL}(\mathrm{ml} / \mathrm{h} / \mathrm{kg}) \dagger$ & $1.184(27.8)$ & $1.059(28.5)$ & $1.112(28.2)$ & $7.158(39.0)$ & $5.812(23.7)$ & $6.401(33.5)$ \\
\hline Vss (dl/kg) † & $1.425(24.1)$ & $1.316(19.7)$ & $1.362(21.8)$ & $1.765(24.8)$ & $1.431(20.5)$ & $1.577(24.7)$ \\
\hline MRT (h) & $122.8(14.2)$ & $129.2(19.0)$ & $126.5(17.1)$ & $27.7(40.9)$ & $25.2(21.2)$ & $26.3(31.4)$ \\
\hline
\end{tabular}

AUC, area under the curve; $\mathrm{CL}$, clearance; \%CV, percent coefficient of variation; FIX, factor IX; IR, incremental recovery; MRT, mean residence time; PK, pharmacokinetic; rIX-FP, recombinant fusion protein linking coagulation factor IX with albumin; $\mathrm{t}_{1 / 2}$, half-life; Vss, volume of distribution at steady-state. * IR is baselinecorrected by FIX activity prior to dose. $+\mathrm{CL}$ and Vss are normalised for body weight. Note: For rIX-FP, for the parameters of $A U C_{0-\infty}, t_{1 / 2}, C L_{1}, V_{s s}$, and MRT, the N for patients $<6$ years of age was 11 , and total $\mathrm{N}$ was therefore 26 . For previous FIX, for the parameters of $A U C_{0-\infty}, t_{1 / 2}, C L, V s S$, and MRT, the N for patients $<6$ years of age was 7 , and total $N$ was therefore 16 . 
three patients were higher than for patients who received routine prophylaxis prior to study entry.

Three patients ( 3,5 and 6 years) had target joints prior to study entry while on routine prophylaxis with previous FIX product. While on weekly prophylaxis with rIX-FP, the target joints resolved in all patients. Two patients had an AsBR of 0.0, and the third patient had an AsBR of 0.99 with no spontaneous bleeds in the target joint in 12 months during the study.

\section{FIX trough levels and rIX-FP consumption during prophylactic regimens}

All patients maintained a weekly routine prophylactic regimen throughout the study, and were compliant with the weekly regimen. The mean weekly consumption of rIX-FP (47 IU/kg) was more than $50 \%$ lower than the weekly consumption for routine prophylaxis with standard FIX products prior to study entry (107 $\mathrm{IU} / \mathrm{kg}$ ). Patients on a weekly dose of 35-50 IU/kg rIX-FP had a median trough of $13.4 \mathrm{IU} / \mathrm{dl}$ FIX activity (IQR 10.3-17.5 IU/dl).

\section{Treatment of bleeding episodes}

A total of 106 bleeding episodes were treated with rIX-FP during the study; $88.7 \%$ of these episodes were successfully treated with one infusion of rIX-FP ( Table 4). The probability of success, defined as requiring $\leq 2$ injections to achieve haemostasis, was $97.2 \%$ (95\% CI: 92.0, 99.0). Similar treatment efficacy was observed in patients in both younger and older age groups, regardless of the cause (spontaneous, trauma-induced or unknown) or location (joint, muscle or other) of the bleeding episodes. Haemostatic effi- cacy of rIX-FP in the treatment of bleeding episodes was rated by investigators as excellent or good for $96.2 \%$ of bleeding episodes.

\section{Surgery}

Two patients had surgery for tooth extractions during the study. One patient ( 8 years) had four teeth extracted due to an abscess, and another patient ( 9 years) had two teeth extracted. The patients received a single dose of 40-50 IU/kg rIX-FP prior to surgery, did not need additional doses during surgery and resumed routine prophylaxis (50-60 IU/kg/week) within a week. One patient had an additional maintenance dose of 32 IU/kg rIX-FP 5 days following surgery. Following surgery, there were no haematomas or bleeding episodes, and treatment was rated as good or excellent. There were an additional five minor surgical procedures (3 cavity fillings, root canal, dental plate insertion) conducted during the study; no additional doses of rIX-FP other than routine prophylaxis were given prior to the procedures and no additional treatments were needed following the procedures.

\section{Safety}

There were 1604 injections of rIX-FP administered during the study with a mean of 61.9 EDs per patient, with 55.3 and 67.3 EDs in younger and older age groups, respectively. No inhibitors against FIX were detected in any patient receiving rIX-FP, and no non-neutralising antibodies against rIX-FP or CHO host cell proteins were detected.

A total of 152 treatment-emergent AEs were reported in 26 (96.3\%) patients. Most treatment-emergent AEs were of mild or

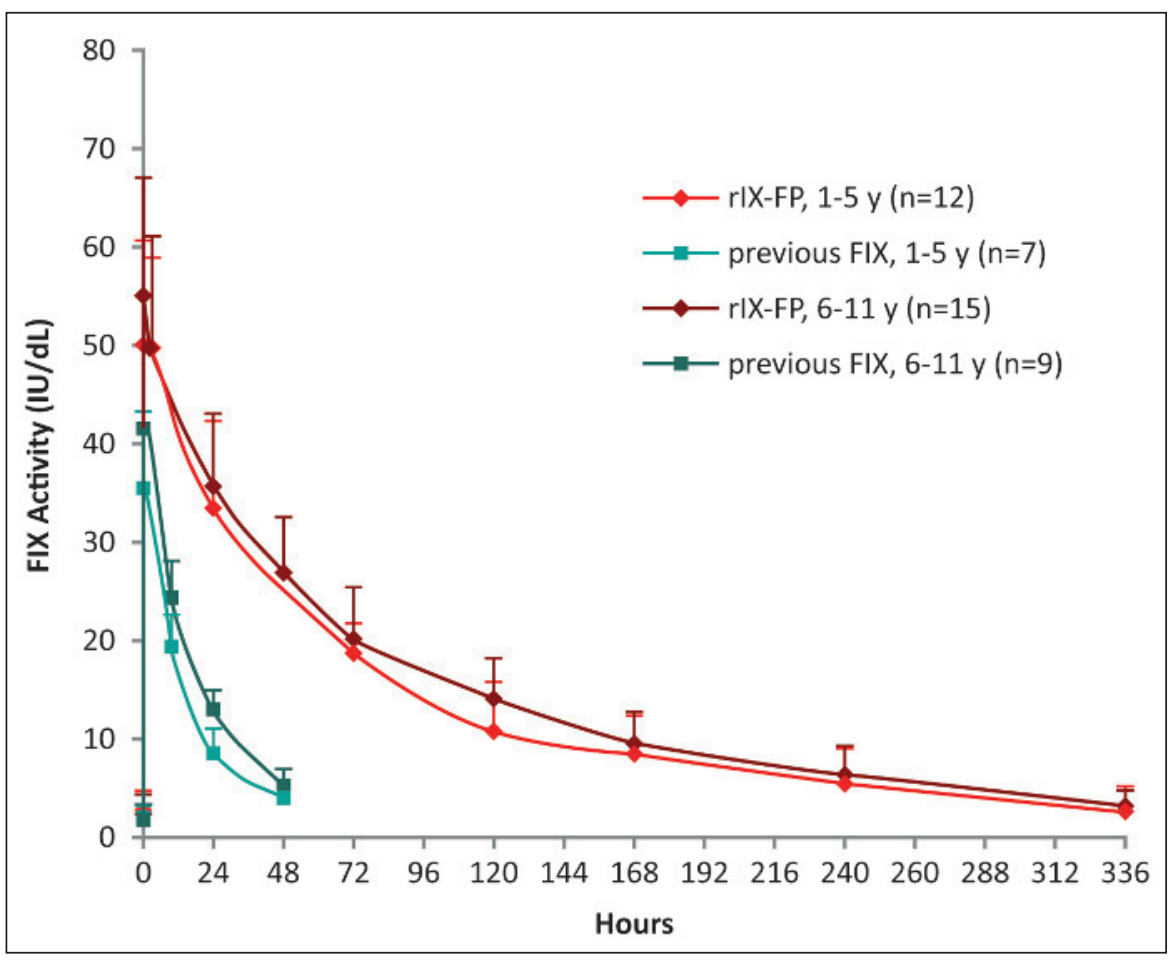

Figure 2: FIX activity after injection of a single dose of rIX-FP or previous FIX product (PK population). Mean baselineuncorrected FIX activity after administration of a single infusion of $50 \mathrm{lU} / \mathrm{kg}$ of rIX-FP or $50 \mathrm{lU} / \mathrm{kg}$ previous FIX product (pdFIX or rFIX). FIX activity was measured in IU/dl. Vertical bars represent standard deviation. FIX, factor IX; pdFIX, plasmaderived FIX; PK, pharmacokinetic; rFIX, recombinant FIX; rIX-FP, recombinant fusion protein linking coagulation factor IX with albumin. 
Table 4: Annualised bleeding rates of children receiving onceweekly prophylaxis with rIX-FP.

\begin{tabular}{|c|c|c|c|}
\hline & $\begin{array}{l}<6 \text { years } \\
(\mathrm{N}=12)\end{array}$ & $\begin{array}{l}6-11 \text { years } \\
(\mathrm{N}=15)\end{array}$ & $\begin{array}{l}\text { Total } \\
(\mathrm{N}=27)\end{array}$ \\
\hline $\begin{array}{l}\text { Number of patients } \\
\text { with } \geq 1 \text { sponta- } \\
\text { neous bleeding epi- } \\
\text { sode, } n(\%)^{*}\end{array}$ & $4(33.3)$ & $9(60.0)$ & $13(48.1)$ \\
\hline \multicolumn{4}{|l|}{ AsBR $†$} \\
\hline Median (IQR) & $0.00(0.00,0.00)$ & $0.78(0.00,1.99)$ & $0.00(0.00,0.91)$ \\
\hline $\begin{array}{l}\text { Estimated rate } \\
(95 \% \mathrm{Cl}) \ddagger\end{array}$ & $0.09(0.01,0.58)$ & $0.86(0.49,1.51)$ & $0.56(0.32,1.00)$ \\
\hline \multicolumn{4}{|l|}{ Joint ABR † } \\
\hline Median (IQR) & $0.50(0.00,1.45)$ & $1.13(0.00,2.36)$ & $0.99(0.00,2.33)$ \\
\hline $\begin{array}{l}\text { Estimated rate } \\
(95 \% \mathrm{Cl}) \neq\end{array}$ & $1.18(0.50,2.82)$ & $1.60(0.91,2.84)$ & $1.44(0.89,2.33)$ \\
\hline \multicolumn{4}{|l|}{ Total ABR † } \\
\hline Median (IQR) & $2.64(2.00,6.48)$ & $3.39(0.76,5.91)$ & $3.12(0.91,5.91)$ \\
\hline $\begin{array}{l}\text { Estimated rate } \\
(95 \% \mathrm{Cl}) \ddagger\end{array}$ & $4.09(2.66,6.28)$ & $3.44(2.13,5.54)$ & $3.69(2.65,5.13)$ \\
\hline \multicolumn{4}{|l|}{ Time on study (days) } \\
\hline Median (IQR) & $\begin{array}{l}356 \text { (313.0, } \\
373.5)\end{array}$ & $\begin{array}{l}456 \text { (361.0, } \\
492.0)\end{array}$ & $\begin{array}{l}382(330.0 \\
469.0)\end{array}$ \\
\hline
\end{tabular}

$A B R$, annualised bleeding rate; $A s B R$, annualised spontaneous bleeding rate; $\mathrm{Cl}$, confidence interval; IQR, interquartile range. The median (IQR) rIX-FP dose during the once-weekly prophylaxis regimen was $47.2 \mathrm{IU} / \mathrm{kg}(40.6$, 55.8). * Number of patients with $\geq 1$ spontaneous bleeding episode during the course of the study. $T$ The ABR was defined as: 365.25 * (number of bleeding episodes) / (number of days in the observed treatment period of interest). $¥$ The estimated rate was calculated assuming a Poisson distribution.

moderate severity (126 mild, 23 moderate, 3 severe). The most frequently reported treatment-emergent AEs were fever (14 events in 9 [33.3\%] patients), contusions (9 events in 5 [18.5\%] patients), nasopharyngitis (6 events in 4 [14.8\%] patients), arthralgia (5 events in 4 [14.8\%] patients), cough (4 events in 4 [14.8\%] patients), bronchitis and ear infection (each: 4 events in 3 [11.1\%] patients), and gastroenteritis and head injury (each: 3 events in 3 [11.1\%] patients). No AEs were considered by the physician to be possibly related to rIX-FP. No patient chose to discontinue treatment after experiencing an $\mathrm{AE}$ or for any other reason.

There were a total of 48 (4.0\%) injection site reactions reported by five patients; all were reported as very slight or slight reactions during home administration of rIX-FP. Four (14.8\%) patients reported six serious adverse events (SAEs), all were associated with accidental injuries. Four patients were hospitalised for a forearm fracture (surgery), deep laceration of the tongue (stitches), groin pain (overnight observation) and head trauma (overnight observation). One patient was hospitalised twice for arthralgia due to bleeding in the hip joint caused by falling on ice. Both of these
Table 5: Summary of mild and moderate bleeding episodes and haemostatic response to rIX-FP.

\begin{tabular}{|c|c|c|c|}
\hline & $<6$ years & 6-11 years & Overall \\
\hline Number of patients & 12 & 15 & 27 \\
\hline $\begin{array}{l}\text { Number of patients with bleed- } \\
\text { ing episodes requiring treatment }\end{array}$ & 11 & 12 & 23 \\
\hline $\begin{array}{l}\text { Number of bleeding episodes } \\
\text { requiring treatment }\end{array}$ & 45 & 61 & 106 \\
\hline \multicolumn{4}{|l|}{ Type of bleeding episode, n (\%) } \\
\hline Spontaneous & $1(2.2)$ & $15(24.6)$ & $16(15.1)$ \\
\hline Traumatic & $38(84.4)$ & $35(57.4)$ & $73(68.9)$ \\
\hline Post-surgery & 0 & $1(1.6)$ & $1(0.9)$ \\
\hline Unknown & $6(13.3)$ & $10(16.4)$ & $16(15.1)$ \\
\hline \multicolumn{4}{|c|}{ Injections to treat a bleeding episode, $\mathrm{n}(\%)$} \\
\hline 1 injection & $40(88.9)$ & $54(88.5)$ & $94(88.7)$ \\
\hline 2 injections & $5(11.1)$ & $4(6.6)$ & $9(8.5)$ \\
\hline$>2$ injections & 0 & $3(4.9)$ & $3(2.8)$ \\
\hline 1 or 2 injections & $45(100.0)$ & $58(95.1)$ & $103(97.2)$ \\
\hline Probability of success ${ }^{*}$, $(95 \% \mathrm{Cl})$ & NC & $\begin{array}{l}95.1 \\
(86.7,98.3)\end{array}$ & $\begin{array}{l}97.2 \\
(92.0,99.0)\end{array}$ \\
\hline $\begin{array}{l}\text { Number of minor/ moderate } \\
\text { bleeding episodes requiring } \\
\text { treatment }\end{array}$ & 43 & 61 & 104 \\
\hline \multicolumn{4}{|l|}{ Haemostatic response $\dagger, \mathrm{n}(\%)$} \\
\hline Excellent & $36(83.7)$ & $42(68.9)$ & $78(75.0)$ \\
\hline Good & $6(14.0)$ & $16(26.2)$ & $22(21.2)$ \\
\hline Moderate & 0 & $1(1.6)$ & $1(1.0)$ \\
\hline Poor/No Response & 0 & 0 & 0 \\
\hline Missing & $1(2.3)$ & $2(3.3)$ & $3(2.9)$ \\
\hline
\end{tabular}

$\mathrm{Cl}$, confidence interval; $\mathrm{NC}$, not calculated. * Probability of success is derived from a repeated measures model (expressed as percentages) and is defined as the probability of achieving haemostasis with 1 or 2 injections. $†$ Haemostatic response was evaluated by the physician. Definitions of haemostatic response for mild to moderate bleeding episodes: Excellent - pain relief and/or unequivocal improvement in objective signs of bleeding at approximately 24 hours after the first infusion and no additional infusions required in order to achieve haemostasis; Good - definite pain relief and/or improvement in signs of bleeding at approximately 24 hours after the first infusion, but required a second infusion in order to achieve haemostasis; Moderate - probable or slight beneficial effect at approximately 24 hours after the first infusion, and required more than 2 injections to achieve haemostasis; Poor/no response - no improvement or worsened at approximately 24 hours after the first infusion and additional haemostatic intervention required with other FIX product or plasma to achieve haemostasis.

bleeding episodes were also considered major bleeds, and resolved after treatment with rIX-FP. All SAEs were considered by the treating physician to be unrelated to rIX-FP. There were no reports of thromboembolic events, anaphylaxis or life-threatening treatment-emergent AEs. 


\section{Discussion}

Results from this prospective phase 3 study in 27 children demonstrate that rIX-FP is a safe and clinically effective FIX replacement product with an improved PK profile supporting a prophylactic dosing interval up to seven days while maintaining FIX trough levels above the target of $1 \mathrm{IU} / \mathrm{dl}$ commonly recommended for prophylaxis according to current treatment guidelines (2).

Weekly prophylaxis with rIX-FP was highly effective in bleeding prevention, as demonstrated by the median AsBR of 0.0. The bleeding episodes in this study were characteristic of those occurring in this young patient population with severe or moderately severe haemophilia B. The majority (68.9\%) of bleeding episodes reported were traumatic, which is to be expected in children due to their high levels of activity.

The total ABR reported in this study was higher than for the adult population (14), in which the proportion of reported bleeds that were traumatic was much lower (39.1\%). The higher total ABR in children likely reflects the higher level of activity in this young patient population. In the older age group, $67 \%$ of patients played sports on a weekly basis, including soccer, which is associated with an increased relative risk of bleeding in children with moderate and severe haemophilia (26). In addition, several previously on-demand patients with a high bleeding rate prior to study entry were enrolled in the study. It is possible that symptoms such as pain or swelling may have been perceived as bleeding episodes by caregivers, particularly in young patients. While nasal bleeds do not generally require FIX replacement therapy in haemophilia patients, many nasal and oral bleeding episodes were treated by caregivers, and hence included in the calculation of ABR.

In addition to effective weekly prophylaxis, patients who had received on-demand only treatment prior to study entry showed a marked reduction in ABR while on prophylaxis with rIX-FP. There were also three patients with target joints at study entry; all target joints were resolved by the end of the treatment period. These pa-

\section{Members of the PROLONG-9FP Investigator Study Group}

Maria-Teresa Álvarez-Roman, Hospital Universitario La Paz, Madrid, Spain; Anthony Chan, McMaster Children's Hospital, Hamilton, ON, Canada; Christopher Barnes, The Royal Children's Hospital, Melbourne, Australia; Jan Blatny, Children's University Hospital, Masaryk University, Brno, Czech Republic; Sandrine Meunier, Groupement Hospitalier Universitaire Est - Hôpital Cardiologique, Bron, Lyon, France; Vladimir Komrska, University Hospital Motol, Prague, Czech Republic; Hans-Juergen Laws, Department of Pediatric Oncology, Hematology and Clinical Immunology, University, Duesseldorf, Germany; Massimo Morfini, Associazione Italiana Centri Emofilici, Florence, Italy; Bohumir Blazek, University Hospital Ostrava, Ostrava-Poruba, Czech Republic. tients had been on twice weekly prophylaxis prior to study entry. While on weekly prophylaxis with rIX-FP, there were no spontaneous bleeds in the target joint in any of these patients.

Measures of haemostatic efficacy indicated that rIX-FP provides rapid and complete control of bleeding episodes with one or two injections, with a success rate of $97.2 \%$. In particular, $88.7 \%$ of bleeds were controlled with one infusion, which compares favourably to results reported for both pdFIX and rFIX products, including extended half-life FIX products $(3,15-17)$.

rIX-FP demonstrated improved PK parameters over all standard FIX products $(3,15,18)$ with an extended terminal half-life of 91.4 h. Other PK parameters, such as AUC (4894 IU.h/dl), clearance $(1.11 \mathrm{ml} / \mathrm{h} / \mathrm{kg})$ and incremental recovery $(1.01 \mathrm{IU} / \mathrm{dl}$ per IU/ $\mathrm{kg}$ ) were also improved as compared to standard FIX products. Incremental recovery and clearance are slightly lower than that seen in adults; however, trough FIX activity in children remains above 5 $\mathrm{IU} / \mathrm{dl}$ at day 7 and above $2 \mathrm{IU} / \mathrm{dl}$ at day 14 following a single dose of $50 \mathrm{IU} / \mathrm{kg}$ rIX-FP. The median rIX-FP dose of $47 \mathrm{IU} / \mathrm{kg}$ for a seven-day prophylactic regimen provided a median FIX activity trough of $13.4 \mathrm{IU} / \mathrm{dl}$. While only a seven-day prophylaxis interval was examined in this study, the PK parameters of rIX-FP suggest that a longer treatment interval may be acceptable for some children. The PK parameters of rIX-FP may enable individualised dosing, such that patients may have a longer interval or higher trough FIX activity level, and should allow children with severe and moderate haemophilia B to experience the benefit of a mild bleeding phenotype.

The longer interval between treatments provided by rIX-FP gives physicians and caregivers more options to consider when weighing the risks and benefits of inserting a central venous access catheter for dosing FIX replacement therapy. Indeed, the efficacy of rIX-FP over the longer treatment interval translates to a reduced number of injections for patients compared with standard rFIX and pdFIX products, and may reduce the burden of disease management for patients, caregivers, and healthcare providers.

Venous access in young children can be a problem to achieve adequate prophylaxis with standard FIX products which require dosing two to three times a week, leading to insertion of a port (4). While the most immediate risk related to a port is excessive bleeding during surgical implantation or extraction, the more common complications are blood infection and clotting. Up to half of patients with ports develop blood clots in the vein accessed by the port's main line (19-21). During the screening period of this study, a two-year-old patient's internal central venous access device clogged. The port was removed prior to the first rIX-FP treatment and was not replaced; the patient was treated with rIX-FP successfully throughout the study without a replacement port. We predict that the necessity of a central venous access device may be very limited due to the extended dose interval of prophylaxis achieved with rIX-FP.

The prolonged half-life, enhanced exposure, and reduced clearance of rIX-FP in young children suggests that patients with severe or moderate haemophilia $B$ may be able to achieve a bleeding phenotype of mild haemophilia $B$ on a routine weekly prophylactic regimen. Low AsBRs, effective treatment of bleeding episodes, and 
clinical improvement of target joints suggests that weekly routine prophylaxis with rIX-FP may provide an improved option for the prevention and treatment of bleeding episodes in children with haemophilia B. Following completion of this study, 24 patients chose to continue treatment with rIX-FP in the extension study (Clinicaltrials.gov number NCT02053792). In addition, approximately $50 \%$ have switched to a longer treatment interval of once every 10 or 14 days with $75 \mathrm{IU} / \mathrm{kg}$ of rIX-FP. Longer treatment intervals of up to 14 days are currently being investigated in children with severe and moderately severe haemophilia B which will continue to provide further clinical efficacy and safety data, in addition to the investigation of rIX-FP in previously untreated paediatric haemophilia $\mathrm{B}$ patients and in perioperative management.

\section{Acknowledgements}

The authors thank all of the patients and their families who contributed to this study, and all of the participating research nurses and data coordinators; the Independent Data Monitoring Committee members: Barbara Konkle (chairperson), Puget Sound Blood Center, Seattle, WA, USA; Mike Makris, Royal Hallamshire Hospital, Sheffield, UK; Amy Dunn, Nationwide Children's Hospital, Columbus, OH, USA. The authors thank Annette Feussner, CSL Behring/Germany for central laboratory testing support, Christine Joch and Denise Wolko, CSL Behring/Germany for data analysis, Grace Cole and Amanda Stetka CSL Behring/United States for operational management, and Tharin Limsakun, CSL Behring/United States for PK review.

\section{Author contributions}

Contribution: G. K., H.C., C.M., T.L., S.H., T.C., M.E.M. and J.C. were principal investigators for the trial. I.J. and C.V. were involved in the design of the trial protocol and authored the manuscript and Y.L. was responsible for statistical analysis. The sponsor was responsible for trial operations, including data analysis. All authors contributed to the interpretation of results, preparation and review of the manuscript.

\section{Conflicts of interest}

G.K., H.C., C.M., T.L., S.H., T.C., M.E.M, J.C. and E.S. received research support from CSL Behring to conduct the study. G.K. received honoraria for speaking and/or for consulting from Opko Biologics, Alnylam, Bayer, Pfizer, Novo Nordisk and research grants from BPL, Baxalta, Pfizer, Opko Biologics. H.C. received honoraria for speaking and/or for consulting from CSL Behring, Bayer Healthcare, Baxter Biosciences, LFB, Novo Nordisk and Pfizer. C.M. has received honoraria for speaking and travel support from Baxter, Bayer, Biotest, CSL Behring, Novo Nordisk and Pfizer. T.L. has received honoraria for speaking and/or for consulting from Baxalta, Bayer, Biogen Ipsen, CSL Behring, LFB, Novo Nordisk, Octapharma, Pfizer and Roche. S.H. received research grant and speakers honorarium from Bayer Healthcare $\mathrm{GmbH}$, Baxalta Innovations $\mathrm{GmbH}$, Biotest AG, CSL Behring GmbH, LFB $\mathrm{GmbH}$, Novartis Pharma GmbH, Novo Nordisk Pharma GmbH, Octapharma GmbH and Pfizer Pharma GmbH. M.E.M. received speaker and consulting fees from CSL Behring, Bayer Healthcare,

\section{What is known about this topic?}

- rIX-FP has demonstrated improved pharmacokinetics, with a mean terminal half-life of 102 h, 4.3-fold longer than previous factor IX (FIX) treatment.

- Weekly and 14-day prophylaxis regimens with rIX-FP were well tolerated and provided low bleeding rates and target joint improvement in adolescent and adult haemophilia B patients.

\section{What does this paper add?}

- The results of this study indicate that rIX-FP has a good safety profile and is effective for preventing and treating bleeding episodes in children with haemophilia B with weekly prophylaxis.

- Mean trough levels were maintained above 5 IU/dI FIX activity in children with a weekly prophylactic rIX-FP regimen.

- Weekly prophylaxis with rIX-FP provides annual spontaneous bleeding rates of zero in the majority of children.

Pfizer, Baxalta, Novo Nordisk and Sobi/Biogen Idec. J.C. received honoraria for speaking and/or for consulting from CSL Behring, Baxter Biosciences, Biogen Idec and Novo Nordisk. E.S. received honoraria for speaking and/or for consulting from CSL Behring, Bayer, Baxter/Baxalta, Pfizer, NovoNordisk, Roche, Sobi/Biogen Idec, Biotest, Kedrion, Octapharma and Grifols and received unrestricted research grants from NovoNordisk and Pfizer. C.V., Y.L. and I. J. were employed at CSL Behring.

\section{References}

1. Bolton-Maggs PHB, Pasi KJ. Haemophilias A and B. Lancet 2003; 361: 1801-1809.

2. Srivastava A, Brewer AK, Mauser-Bunschoten EP, et al. Guidelines for the management of hemophilia. Haemophilia 2012; 19: e1-e47.

3. RIXUBIS Package Insert. Available at: http://www.rixubis.com/pdf/rixubis_pi. pdf. Accessed 22nd February 2016.

4. Santagostino E, Mancuso ME. Venous access in haemophilic children: choice and management. Haemophilia 2010; 16: 20-24.

5. Santagostino E, Negrier C, Klamroth R, et al. Safety and pharmacokinetics of a novel recombinant fusion protein linking coagulation factor IX with albumin (rIX-FP) in hemophilia B patients. Blood 2012; 120: 2405-2411.

6. Martinowitz U, Lissitchkov T, Lubetsky A, et al. Results of a phase I/II openlabel, safety and efficacy trial of coagulation factor IX (recombinant), albumin fusion protein in haemophilia B patients. Haemophilia 2015; 21: 784-790.

7. International Conference on Harmonization. Harmonized Tripartite Guideline: Guideline for Good Clinical Practice (E6). ICH. 1996. Available at: http://www. ich.org/fileadmin/Public_Web_Site/ICH_Products/Guidelines/Efficacy/ E6/E6_R1_Guideline.pdf. Accessed February 22, 2016.

8. European Medicines Agency. Committee for medicinal products for human use (CHMP), Guideline in the Clinical Investigation of Recombinant and Human Plasma-Derived Factor IX Products. EMEA. 2009. CHMP/BPWP/144552/2009.

9. Verbruggen B, Novakova I, Wessels $\mathrm{H}$, et al. The Nijmegen modification of the Bethesda assay for factor VIII:C inhibitors: improved specificity and reliability. Thromb Haemost 1995; 73: 247-251.

10. Windyga J, Lissitchkov T, Stasyshyn O, et al. Efficacy and safety of a recombinant factor IX (Bax326) in previously treated patients with severe or moderately severe haemophilia B undergoing surgical or other invasive procedures: a prospective, open-label, uncontrolled, multicentre, phase III study. Haemophilia 2014; 20: 651-658. 
11. Morfini M, Lee M, Messori A. The design and analysis of half-life and recovery studies for factor VIII and factor IX. Factor VIII/Factor IX Scientific and Standardization Committee of the International Society for Thrombosis and Haemostasis. Thromb Haemost 1991; 66: 384-386.

12. Lee M, Morfini M, Schulman S, et al. Scientific and Standardization Committee Communication: The Design and Analysis of Pharmacokinetic Studies of Coagulation Factors. Available at: http://c.ymcdn.com/sites/www.isth.org/re source/group/d4a6f49a-f4ec-450f-9e0f-7be9f0c2ab2e/official_communications/fviiipharmaco.pdf. Accessed February 22, 2016.

13. Rallapalli PM, Kemball-Cook G, Tuddenham EG, et al. An interactive mutation database for human coagulation factor IX provides novel insights into the phenotypes and genetics of hemophilia B. J Thromb Haemost 2013; 11: 1329-1340.

14. Santagostino E, Martinowitz U, Lissitchkov T, et al. Long acting recombinant coagulation factor IX albumin fusion protein (rIX-FP) in hemophilia B: results of a phase 3 trial. Blood 2016;127: 1761-1769.

15. Alprolix Package Insert. Available at: http://www.alprolix.com/pdfs/Prescrib ingInformation.pdf. Accessed 22nd February 2016.

16. Collins PW, Young G, Knobe K, et al. Recombinant long-acting glycoPEGylated factor IX in hemophilia B: a multinational randomized phase 3 trial. Blood 2014; 124: 3880-3886.

17. Roth DA. Human recombinant factor IX: safety and efficacy studies in hemophilia B patients previously treated with plasma-derived factor IX concentrates. Blood 2001; 98: 3600-3606.
18. Benefix Package Insert. Available at: http://labeling.pfizer.com/showlabeling.aspx?id=492. Accessed February 22, 2016.

19. Journeycake JM. Catheter-related deep venous thrombosis in children with hemophilia. Blood 2001; 98: 1727-1731.

20. Price VE, Carcao M, Connolly B, et al. A prospective, longitudinal study of central venous catheter-related deep venous thrombosis in boys with hemophilia. J Thromb Haemost 2004; 2: 737-742.

21. Ranta S, Kalajoki-Helmiö T, Pouttu J, et al. MRI after removal of central venous access device reveals a high number of asymptomatic thromboses in children with haemophilia. Haemophilia 2011; 18: 521-526.

22. Blanchette VS, Key NS, Ljung LR, et al. Definitions in hemophilia: communication from the SSC of the ISTH. J Thromb Haemost 2014; 12: 1935-1939.

23. HGVS. Nomenclature for the description of sequence variations homepage. Available at. Accessed December 10, 2015.

24. Goodeve AC, Reitsma PH, McVey JH. Nomenclature of genetic variants in hemostasis. J Thromb Haemost 2011; 9: 852-855.

25. CDC Hemophilia B Mutation Project (CHBMP) F9 Database. Available at: http://www.cdc.gov/ncbddd/hemophilia/champs.html. Accessed February 22, 2016.

26. Broderick CR, Herbert RD, Latimer J. Association between physical activity and risk of bleeding in children with hemophilia. J Am Med Assoc 2012; 308: 1452-1459.

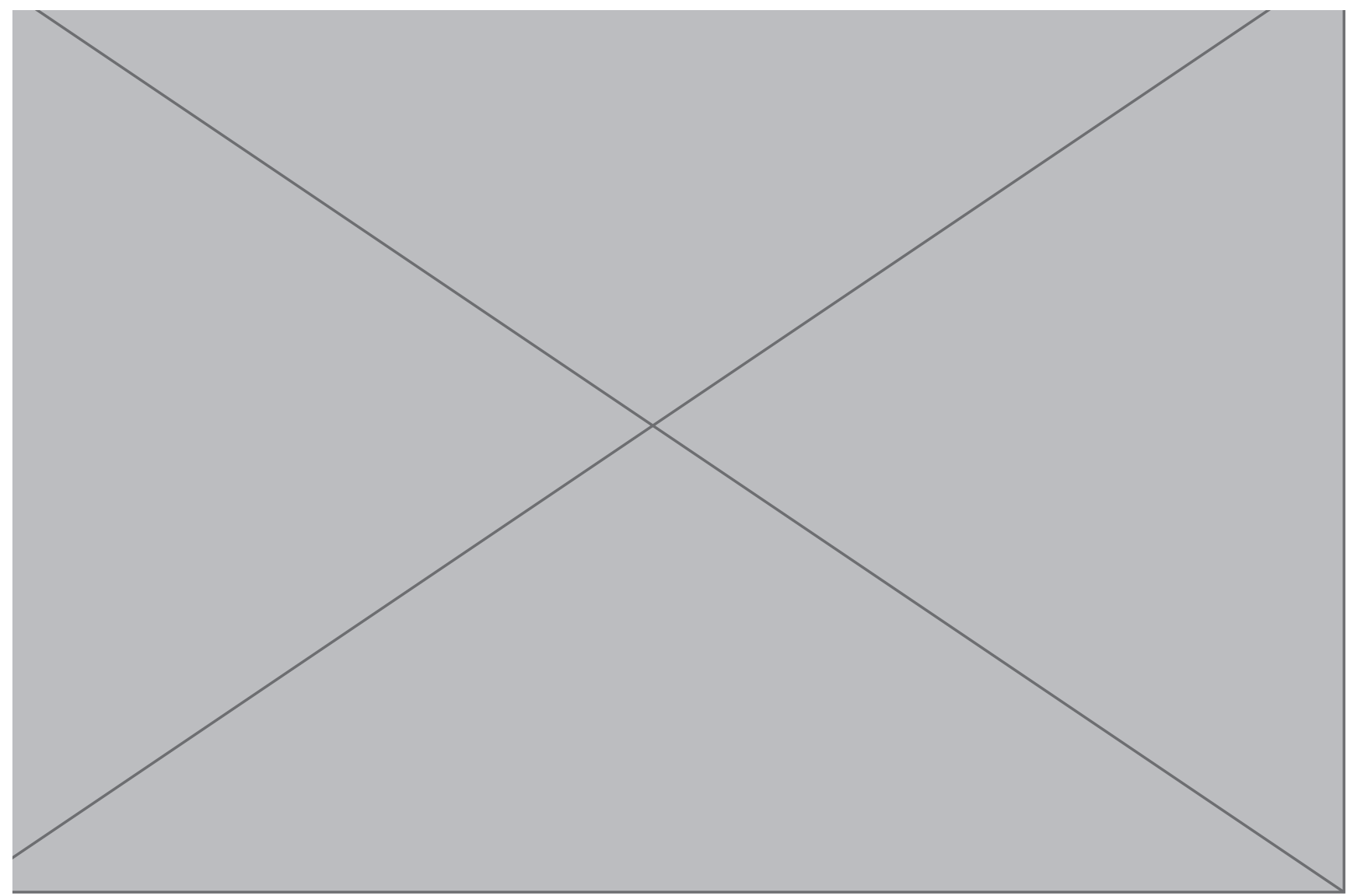
not used for commercial purposes and no modifications or adaptations are made. 